\title{
Endovascular therapy of isolated internal iliac artery aneurysm
}

\author{
Abdullah Ozer ${ }^{1 *}$, Yigit Kilic ${ }^{1}$, Baris Mardin ${ }^{1}$, Koray Akkan $^{2}$, Levent Oktar ${ }^{1}$
}

\begin{abstract}
Isolated internal iliac artery (IIA) aneurysms are relatively uncommon. Isolated IIA aneurysms traditionally have been treated by surgical reconstruction. We report our experience with endovascular treatment of isolated IIA aneurysm.
\end{abstract}

Key words: Iliac Aneurysm; Endovascular Procedures; Vascular Grafting

\section{Introduction}

An isolated aneurysm of the IIA is relatively uncommon and is generally asymptomatic. It is often diagnosed in a late stage and it carries a high rupture risk. We describe our experience of an isolated IIA aneurysm case that we treated with a branched stentgraft, which is in general a rarely performed procedure

\section{Case Report}

A 57-year-old male patient presenting with pain in his right thigh underwent $\mathrm{CT}$ angiography which showed an aneurysmatic dilatation of nearly $40 \mathrm{~mm}$ in a $6 \mathrm{~cm}$ segment of the right common iliac artery to the proximal regions of the iliac arteries. The widest part of the dilatation showed the presence of a nearly 20 $\mathrm{mm}$ thrombus. The left common iliac artery showed an aneurysmatic dilation of a nearly $3 \mathrm{~cm}$ segment reaching proximally, with a maximum diameter of 18 $\mathrm{mm}$ where a $2 \mathrm{~mm}$ thrombus was seen.

Via a right femoral arteriotomy a sheath was placed including a stiff guidewire that was led to the thoracic aorta. A second guidewire was then inserted in a retrograde manner to the terminal aorta and was guided out of the vessel at the left femoral level using a cross-over approach.

The distal end of the stent-graft main body was placed to cover the aneurysm proximally and to appropriately guide the IIA. The guidewire that was taken out of the vessel by a cross-over approach and via a left arteriotomy was used to lead the vascular sheath to the proximal end of the stent-graft at the aortoiliac level.
The distal end of the vascular sheath that was inserted via a left arteriotomy, was placed at the level of the stent-graft main body. The sheath was then used to lead the guidewire through the internal iliac branch of the stent-graft main body.

Via a left femoral approach, the guidewire was led through the internal iliac branch of the stent-graft main body and was placed in the right IIA where the internal iliac limb was positioned.

\section{Discussion}

A systematic review of studies of the autopsy from both North America and Europe showed that the incidence of isolated iliac aneurysms varies between $0.03 \%$ and $0.1 \% 1,2,3$ as compared with $3.8 \%$ for abdominal aortic aneurysms. 3

Because of the deep pelvic location it is difficult to diagnose isolated iliac artery aneurysm on physical examination and it likely accounts for the high incidence of symptomatic or ruptured aneurysms in the past. 4 Today, most of iliac artery aneurysms are usually detected as an incidental finding during diagnostic imaging while searching for other suspected conditions.

Endovascular management has offered the ability to deal with the challenges of deep pelvic structures with minimal morbidity with perioperative mortality rates approaching $0 \% .5,6,7$

Received 28-06-2016 Accepted 01-07-2016 Available Online 15-08-2016

1 Gazi University Faculty of Medicine, Department of Cardiovascular Surgery, Ankara, Turkey

2 Gazi University Faculty of Medicine, Department of Radiology, Anakar, Turkey

* Corresponding Author: Abdullah Ozer E-mail: dr-abdozer@hotmail.com Phone: +90 2025619 


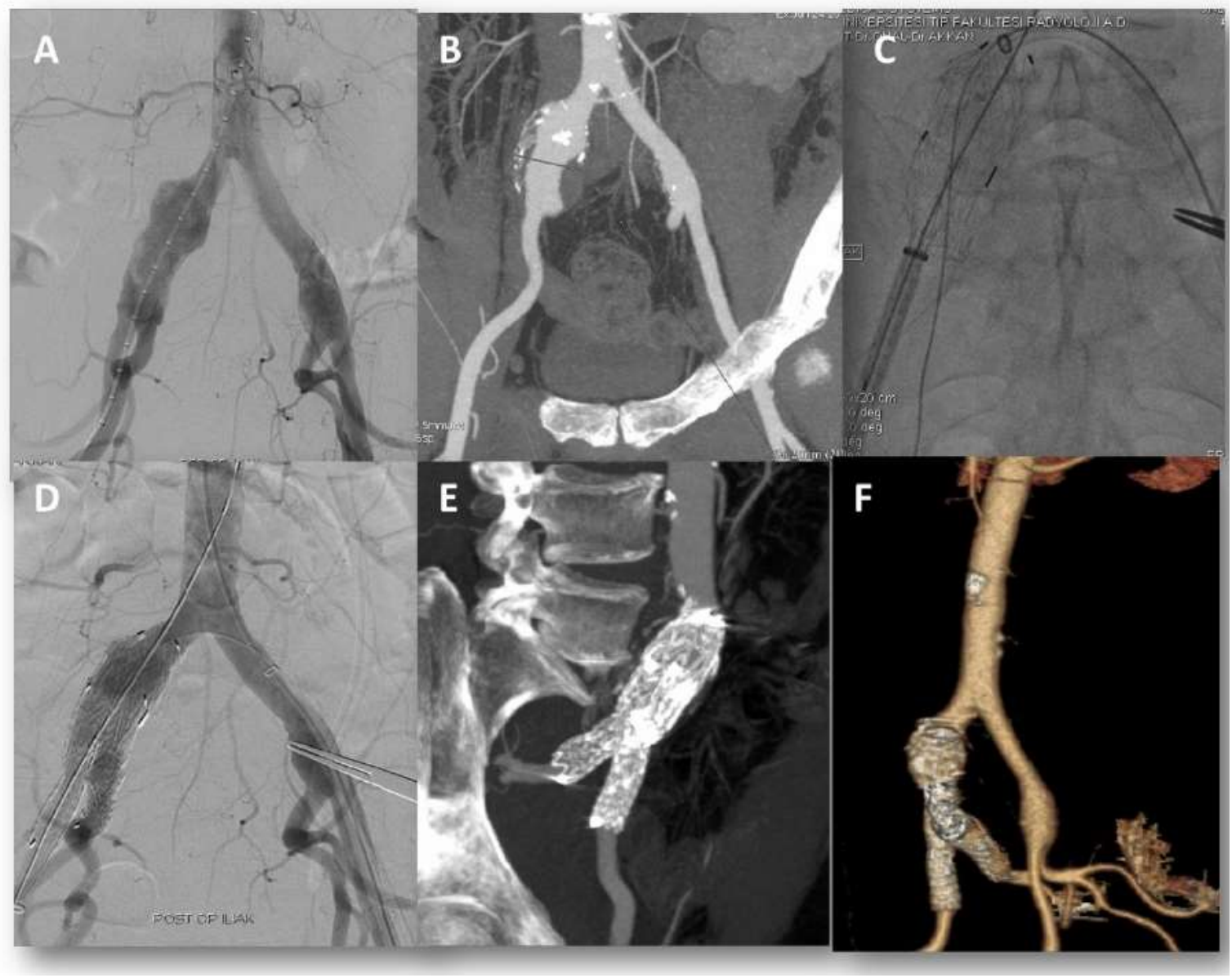

Figure 1: A, B: Aneurysmatic dilatation of nearly $40 \mathrm{~mm}$ in a $6 \mathrm{~cm}$ segment of the right common iliac artery C: Positioning of branched stent-graft D: Postoperative DSA E: Postoperative MIP CT F: Postoperative 3D CT

\section{Conclusion}

Treatment of an isolated IIA aneurysm with a branched stent-graft is a rarely performed procedure with a low morbidity rate and a high success rate, and it led to a symptomatic improvement in our case.

Conflict of Interest: The authors declare no potential conflicts of interest with respect to the research, authorship, and/or publication of this article.

Ethical issues: All Authors declare that Originality of research/article etc... and ethical approval of research, and responsibilities of research against local ethics commission are under the Authors responsibilities. The study was completed due to defined rules by the Local Ethics Commission guidelines and audits.

\section{Aknowledgement: None.}

\section{References}

1. Brunkwall J, Hauksson H, Bengtsson H, Bergqvist D, Takolander R, Bergentz SE. Solitary aneurysms of the iliac arterial system: an estimate of their frequency of occurrence. Journal of vascular surgery. 1989 Oct 31;10(4):381-4.

2. Lucke B, REA MH. STUDIES ON ANEURYSM I. GENERAL STATISTICAL DATA ON ANEURYSM. Journal of the American Medical Association. 1921 Sep 17;77(12):935-40.

3. Kunz R. Aneurysmata bei 35380 Autopsien. Schweiz Med Woehensehr 1980; 110: 142-8

4. Krupski WC, Selzman CH, Floridia R, Strecker PK, Nehler MR, Whitehill TA. Contemporary management of isolated iliac aneurysms. Journal of vascular surgery. $1998 \mathrm{Jul}$ 31;28(1):1-3. 
5. Boules TN, Selzer F, Stanziale SF, Chomic A, Marone LK, Dillavou ED, Makaroun MS. Endovascular management of isolated iliac artery aneurysms. Journal of vascular surgery. 2006 Jul 31;44(1):29-37.

6. Caronno R, Piffaretti G, Tozzi M, Lomazzi C, Rivolta N Laganà D, Carrafiello G, Recaldini C, Castelli P. Endovascular treatment of isolated iliac artery aneurysms. Annals of vascular surgery. $2006 \mathrm{Jul}$ 1;20(4):496-501
7

Fahrni M, Lachat MM, Wildermuth S, Pfammatter T. Endovascular therapeutic options for isolated iliac aneurysms with a working classification. Cardiovascular and interventional radiology. 2003 Sep 1;26(5):443-7.

Copyright (C) 2016 The Author(s); This is an open-access article distributed under the terms of the Creative Commons Attribution License (http://creativecommons.org/licenses/by/4.0), which permits unrestricted use, distribution, and reproduction in any medium, provided the original work is properly cited. All Rights reserved by international journal of Medical Science and Discovery. 